Article

\title{
Anti-Oxidative and Anti-Proliferative Activity on Human Prostate Cancer Cells Lines of the Phenolic Compounds from Corylopsis coreana Uyeki
}

\author{
Manh Heun Kim ${ }^{\dagger}$, Sung Yi Ha ${ }^{\dagger}$, Myeong Hwan Oh, Han Hyuk Kim, So Ra Kim and \\ Min Won Lee * \\ College of Pharmacy, Chung-Ang University, Seoul 156-756, Korea \\ $\dagger$ These authors contributed equally to this work. \\ * Author to whom correspondence should be addressed; E-Mail: mwlee@cau.ac.kr; \\ Tel.: +82-2-820-5602; Fax: +82-2-822-9778.
}

Received: 4 January 2013; in revised form: 20 April 2013 / Accepted: 23 April 2013 /

Published: 24 April 2013

\begin{abstract}
Fifteen phenolic compounds, including three caffeoyl derivatives, four gallotannins, three ellagitannins and five flavonoids, were isolated from an $80 \% \mathrm{MeOH}$ extract of the leaves of Corylopsis coreana Uyeki (Korean winter hazel; CL). The antioxidative activities [1,1-diphenyl-2-picrylhydrazyl (DPPH) radical scavenging activity and xanthine oxidase superoxide scavenging activities (NBT)] and the anti-proliferative activity on human prostate cancer cell lines (DU145 and LNCaP) were also evaluated.
\end{abstract}

Keywords: Corylopsis coreana. Uyeki; 1,1-diphenyl-2-picrylhydazyl radical; superoxide scavenging; LNCaP; DU145

\section{Introduction}

Corylopsis coreana Uyeki (Korean winter hazel; CL) belongs to the Hamamelidaceae or witch hazel family, [1]. CL is cultivated as an ornamental plant in South Korea. Some species of the genus Corylopsis, such as Hamamelis virginiana (witch hazel) have been a folk medicine for the treatment of irritated skin and inflammatory disease [2] and witch hazel bark are widely used in skin care products for sun burn, atopic eczema, etc. [3]. It has been reported that hamamelitannin and simple phenolics were isolated from the bark of witch hazel [4], and their anti-mutagenic activities and anti-cancer 
activities against various forms of cancer were also reported [5]. This paper describes the isolation of compounds from the leaves of $\mathrm{CL}$ and evaluation of its anti-oxidative activities and the anti-proliferation properties of the isolated compounds.

\section{Results and Discussion}

\subsection{Isolation and Identification}

Anti-oxidant activity-guided chromatographic fractionation of leaves of CL afforded fifteen phenolic compounds (Scheme 1 and Table 1). The structures of compounds 1-15 were identified as 3-caffeoylquinic acid methyl ester (1) [6], 4-caffeoylquinic acid (2) [6], 3-caffeoylquinic acid (3) [6], 3-O-galloyl- $\beta$-D-glucopyranoside (4) [7], bergenin (5) [8], nor-bergenin (6) [8], 11-galloylbergenin (7) [9], tellimagrandin I (8) [10], tellimagrandin II (9) [11], casuarinin (10) [12], quercetin (11) [13], quercitrin (12) [14], quercetin 3- $O-\beta$-D-glucuronide (13) [15], datiscetin 3- $O-\beta$-D-rhamnpyranoside (14) [16] and myricetin 3-O- $\beta$-D-rhamnopyranoside (15) [17], respectively (Figure 1), by comparing the spectral (MS, NMR) data with the values reported in the literature.

Scheme 1. Extraction and Isolation of Compounds 1-15 from CL.

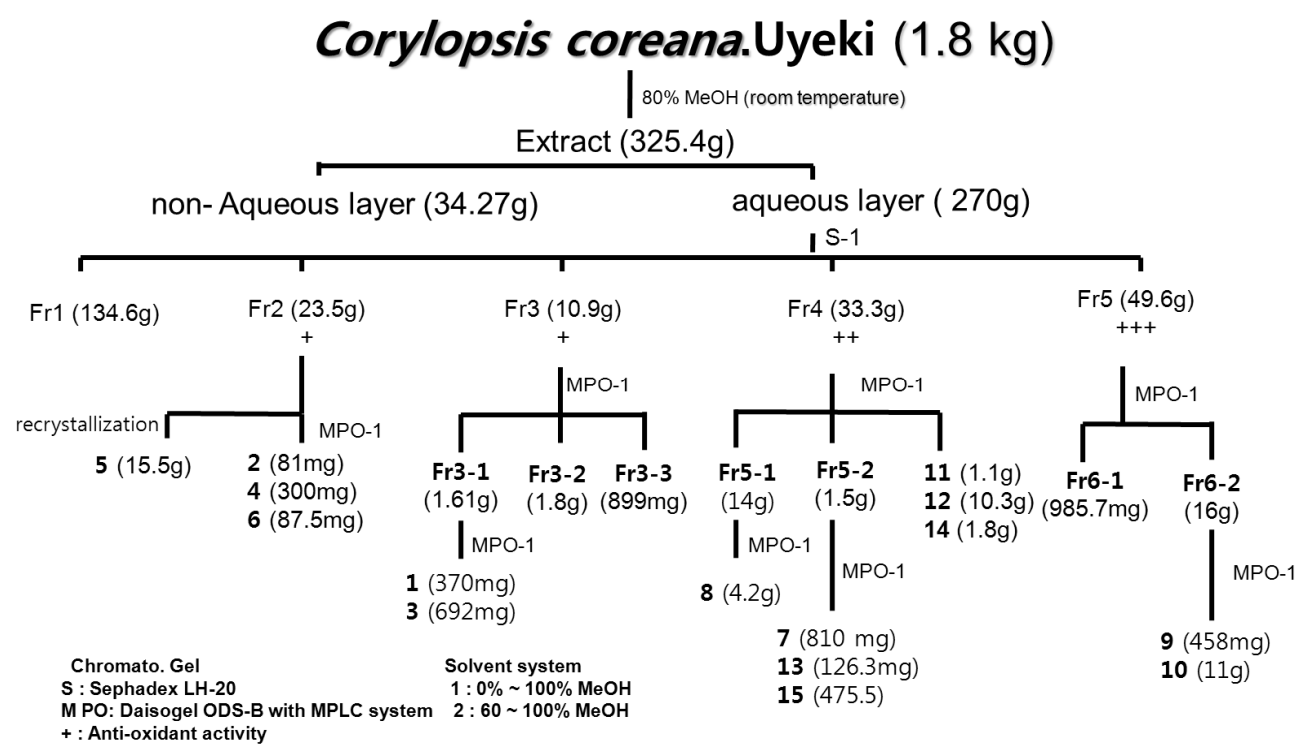

Table 1. DPPH radical scavenging and superoxide scavenging activities of each fraction from CL.

\begin{tabular}{|c|c|c|}
\hline Fractions & $\begin{array}{l}\text { DPPH radical scavenging activity } \\
\qquad \mathrm{SC}_{50}(\mathrm{mg} / \mathrm{mL})\end{array}$ & $\begin{array}{l}\text { Superoxide scavenging activity } \\
\mathrm{SC}_{50}(\mathrm{mg} / \mathrm{mL})\end{array}$ \\
\hline 1 & $>100^{\mathrm{e}}$ & $59.02 \pm 1.07^{\mathrm{d}}$ \\
\hline 2 & $43.16 \pm 0.48^{d}$ & $23.64 \pm 0.92^{c}$ \\
\hline 3 & $20.26 \pm 0.43^{c}$ & $13.35 \pm 1.09^{b}$ \\
\hline 4 & $11.22 \pm 024^{b}$ & $5.89 \pm 0.91^{\mathrm{a}}$ \\
\hline 5 & $5.89 \pm 0.03^{\mathrm{a}}$ & $2.42 \pm 0.73^{\mathrm{a}}$ \\
\hline Ascorbic acid & $6.68 \pm 0.11^{\mathrm{a}}$ & - \\
\hline Allopurinol & - & $1.70 \pm 0.92^{\mathrm{a}}$ \\
\hline
\end{tabular}

Values represent means \pm S.D. of three determinations. Values bearing different superscripts in the same column are significantly different $(p<0.05)$. 
Figure 1. Structures of compounds 1-15 isolated from CL.

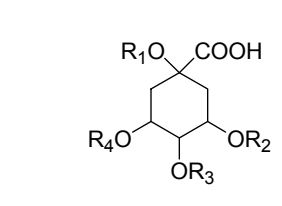

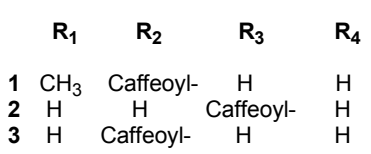

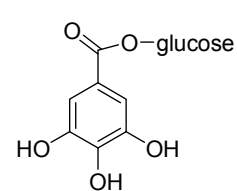

4

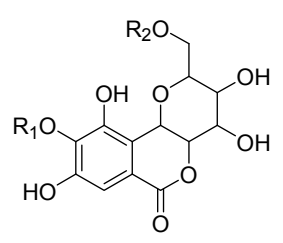

$\begin{array}{llc} & \mathbf{R}_{\mathbf{1}} & \mathbf{R}_{\mathbf{2}} \\ \mathbf{5} & \mathrm{CH}_{3} & \mathrm{H} \\ \mathbf{6} & \mathrm{H} & \mathrm{H} \\ \mathbf{7} & \mathrm{CH}_{3} & \beta-O-\text {-galloyl- }\end{array}$

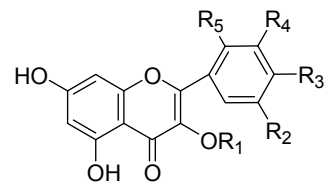

$\begin{array}{lccccc} & \mathbf{R}_{\mathbf{1}} & \mathbf{R}_{\mathbf{2}} & \mathbf{R}_{\mathbf{3}} & \mathbf{R}_{\mathbf{4}} & \mathbf{R}_{\mathbf{5}} \\ \mathbf{1 1} & \mathrm{H} & \mathrm{H} & \mathrm{OH} & \mathrm{OH} & \mathrm{H} \\ \mathbf{1 2} & \text { rhamnose } & \mathrm{H} & \mathrm{OH} & \mathrm{OH} & \mathrm{H} \\ \mathbf{1 3} & \text { glucuronic acid } & \mathrm{H} & \mathrm{OH} & \mathrm{OH} & \mathrm{H} \\ \mathbf{1 4} & \text { rhamnose } & \mathrm{H} & \mathrm{H} & \mathrm{H} & \mathrm{OH} \\ \mathbf{1 5} & \text { rhamnose } & \mathrm{OH} & \mathrm{OH} & \mathrm{OH} & \mathrm{H}\end{array}$

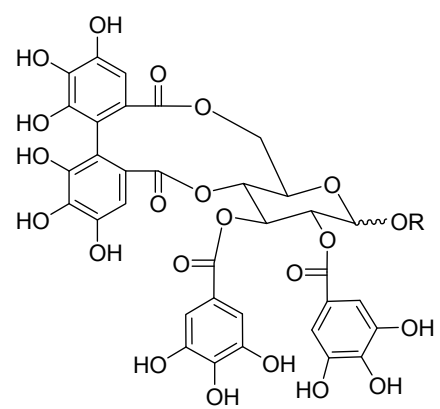

$\mathbf{R}$

$\begin{array}{lc}8 & \mathrm{H} \\ 9 & \beta-O-g a l l o y l-\end{array}$

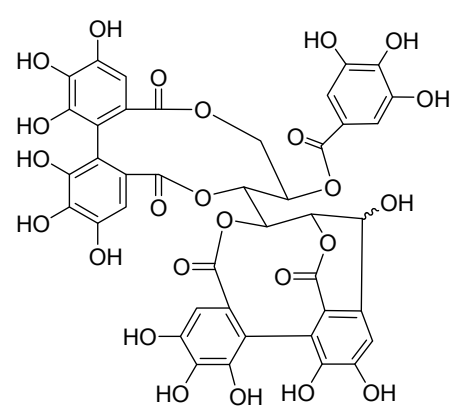

10

\subsection{Anti-Oxidative Activity (DPPH Radical, Superoxide Scavenging Activity)}

Oxidative damage appears to be related to various diseases and inflammation conditions $[18,19]$. Polyphenols reduce oxidative stress and provide activity associated with anti-cancer, anti-allergic, and anti-inflammatory effects [20]. In order to evaluate the anti-oxidative activities of the compounds 1-10 from CL, their DPPH radical [21] and superoxide scavenging activities were tested [22]. Among the compounds 1-10 from CL, 8, 9 and 10 showed more potent free radical scavenging activity than ascorbic acid $\left(\mathrm{SC}_{50}=8.22 \pm 0.64 \mu \mathrm{M}\right)$, with $\mathrm{SC}_{50}$ values of $3.12 \pm 0.05,2.97 \pm 0.04$ and $3.29 \pm 0.26 \mu \mathrm{M}$ $(p<0.05)$ (Table 1), due to presence of a galloyl groups plus an HHDP group in their structures, which plays an important role in anti-oxidation by donating hydrogen atoms to active free radicals [23]. In addition, 8, 9 and 10 also showed more potent superoxide scavenging activity than allopurinol $\left(\mathrm{SC}_{50}=\right.$ $2.39 \pm 0.09 \mu \mathrm{M})$, with $\mathrm{SC}_{50}$ values of $0.29 \pm 0.69,0.09 \pm 0.02$ and $0.16 \pm 0.02 \mu \mathrm{M}(p<0.05)$ (Table 2).

\subsection{Cell Viability and Inhibition of Cancer Cell Proliferation}

The cell viability was measured using the MTT assay (Figure 2), which is based on the mitochondria-dependent reduction of MTT to formazan [24]. In order to evaluate the anti-proliferation activities of the compounds 1-15 from CL, cell viability were tested on DU145 and LNCaP prostate cancer cells. The anti-proliferative effects of hydrolysable tannins in sarcoma cells and HeLa cells were reported [25] and the functional groups the hydrolysable tannins are also important factors determining their anti-proliferation activity [26]. Among the ellagitannins 8-10, compound 10 showed 
higher androgen sensitive anti-proliferation activity, suggesting the importance of the HHDP group. Since 9 was more potent than 10, the presence of both HHDP and galloyl groups might be necessary. Compound $\mathbf{9}$ was also more potent than $\mathbf{8}$, suggesting the importance of a galloyl group in the C-1 position [27]. The compounds $\mathbf{8}, \mathbf{9}$ and $\mathbf{1 0}$ inhibited the proliferation of both DU145 and LNCaP prostate cancer cells (Table 3 ).

Table 2. DPPH radical scavenging and superoxide scavenging activities of each compounds 1-15 from CL.

\begin{tabular}{ccc}
\hline Compounds & $\begin{array}{c}\text { DPPH radical scavenging activity } \\
\mathbf{S C}_{\mathbf{5 0}}(\boldsymbol{\mu} \mathbf{M} / \mathbf{m L})\end{array}$ & $\begin{array}{c}\text { Superoxide scavenging activity } \\
\mathbf{S C}_{\mathbf{5 0}}(\boldsymbol{\mu} \mathbf{M} / \mathbf{m L} \mathbf{L})\end{array}$ \\
\hline $\mathbf{1}$ & $23.05 \pm 0.92^{\mathrm{d}, \mathrm{e}}$ & $13.47 \pm 0.05^{\mathrm{b}}$ \\
$\mathbf{2}$ & $51.49 \pm 0.77^{\mathrm{g}}$ & $11.71 \pm 0.36^{\mathrm{b}}$ \\
$\mathbf{3}$ & $21.65 \pm 0.72^{\mathrm{d}, \mathrm{e}}$ & $22.68 \pm 0.35^{\mathrm{d}}$ \\
$\mathbf{4}$ & $13.41 \pm 0.10^{\mathrm{c}}$ & $10.81 \pm 0.07^{\mathrm{c}}$ \\
$\mathbf{5}$ & $>100^{\mathrm{i}}$ & $>100^{\mathrm{e}}$ \\
$\mathbf{6}$ & $36.34 \pm 0.93^{\mathrm{f}}$ & $13.68 \pm 0.74^{\mathrm{b}, \mathrm{c}}$ \\
$\mathbf{7}$ & $21.63 \pm 0.70^{\mathrm{e}}$ & $3.85 \pm 0.03^{\mathrm{a}}$ \\
$\mathbf{8}$ & $3.12 \pm 0.05^{\mathrm{a}}$ & $0.29 \pm 0.69^{\mathrm{a}}$ \\
$\mathbf{9}$ & $2.97 \pm 0.04^{\mathrm{a}}$ & $0.09 \pm 0.02^{\mathrm{a}}$ \\
$\mathbf{1 0}$ & $3.29 \pm 0.26^{\mathrm{a}}$ & $0.16 \pm 0.02^{\mathrm{a}}$ \\
$\mathbf{1 1}$ & $49.09 \pm 0.92^{\mathrm{g}}$ & $12.18 \pm 0.14^{\mathrm{b}, \mathrm{c}}$ \\
$\mathbf{1 2}$ & $60.96 \pm 0.70^{\mathrm{h}}$ & $12.43 \pm 0.24^{\mathrm{b}, \mathrm{c}}$ \\
$\mathbf{1 3}$ & $17.06 \pm 0.15^{\mathrm{d}}$ & $3.17 \pm 0.78^{\mathrm{a}}$ \\
$\mathbf{1 4}$ & $36.87 \pm 0.44^{\mathrm{f}}$ & $2.59 \pm 0.75^{\mathrm{a}}$ \\
$\mathbf{1 5}$ & $19.45 \pm 0.05^{\mathrm{d}, \mathrm{e}}$ & $1.45 \pm 0.24^{\mathrm{a}}$ \\
Ascorbic acid & $8.22 \pm 0.64^{\mathrm{b}}$ & - \\
Allopurinol & - & $2.39 \pm 0.09^{\mathrm{a}}$ \\
\hline
\end{tabular}

Values represent means \pm S.D. of three determinations. Values bearing different superscripts in the same column are significantly different $(p<0.05)$.

Figure 2. Cell viability of compounds 1-15 from CL on RAW 264.7 cell lines.

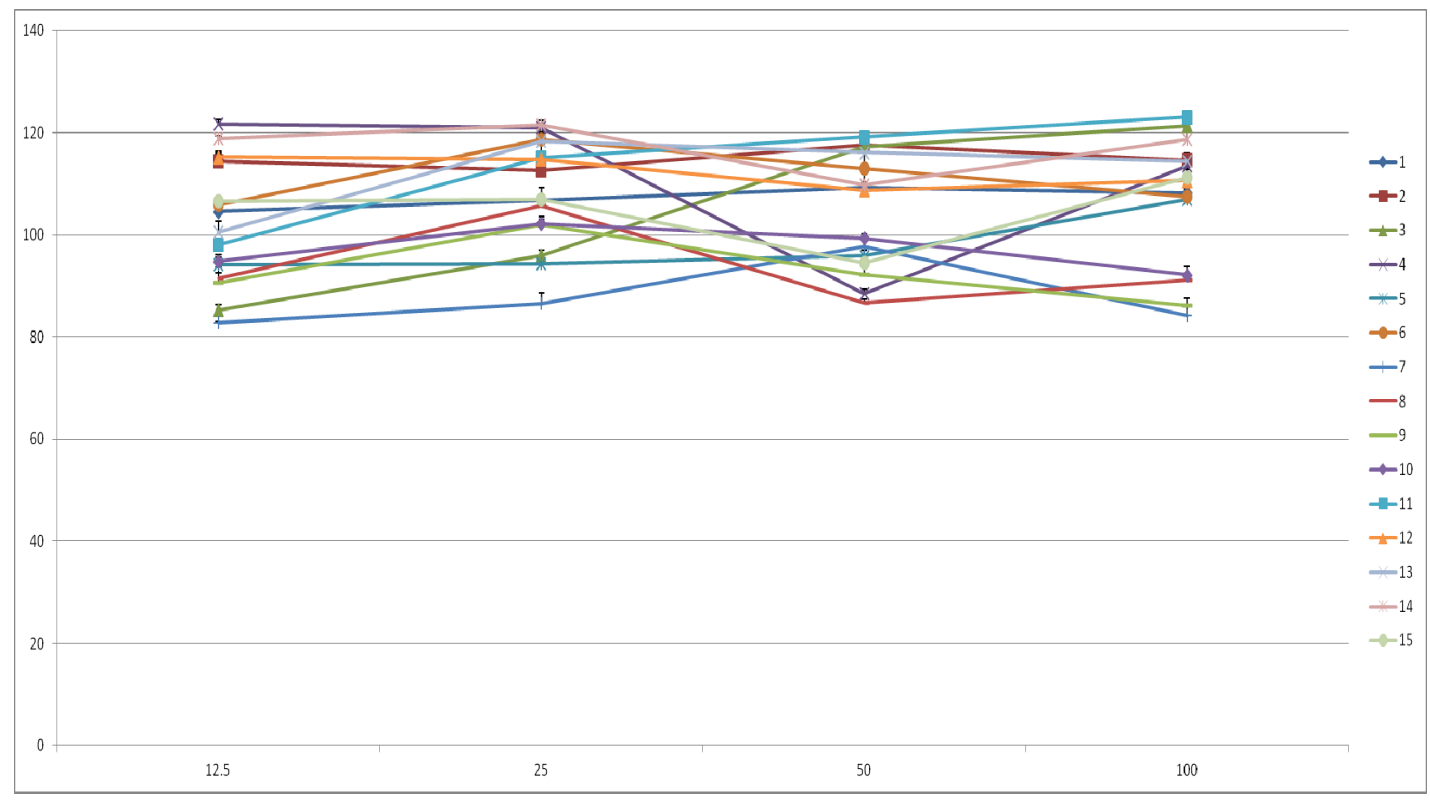


Table 3. Anti-proliferative effects of compounds 1-15 from CL on LNCaP and DU145 cancer cell lines.

\begin{tabular}{ccc}
\hline Compounds & $\begin{array}{c}\text { Anti-proliferative activity of LNCaP } \\
\text { cancer cell line } \mathbf{I C}_{\mathbf{5 0}}(\boldsymbol{\mu M} \mathbf{M L})\end{array}$ & $\begin{array}{c}\text { Anti-proliferative activity of Du-145 } \\
\text { cancer cell line } \mathbf{I C}_{\mathbf{5 0}}(\boldsymbol{\mu M} \mathbf{M} \mathbf{m L})\end{array}$ \\
\hline $\mathbf{1}$ & $>100^{\mathrm{c}}$ & $>100^{\mathrm{a}}$ \\
$\mathbf{2}$ & $>100^{\mathrm{c}}$ & $>100^{\mathrm{a}}$ \\
$\mathbf{3}$ & $>100^{\mathrm{c}}$ & $>100^{\mathrm{a}}$ \\
$\mathbf{4}$ & $>100^{\mathrm{c}}$ & $>100^{\mathrm{a}}$ \\
$\mathbf{5}$ & $>100^{\mathrm{c}}$ & $>100^{\mathrm{a}}$ \\
$\mathbf{6}$ & $>100^{\mathrm{c}}$ & $>100^{\mathrm{a}}$ \\
$\mathbf{7}$ & $>100^{\mathrm{c}}$ & $>100^{\mathrm{a}}$ \\
$\mathbf{8}$ & $66.35 \pm 1.44^{\mathrm{b}}$ & $>100^{\mathrm{a}}$ \\
$\mathbf{9}$ & $43.08 \pm 0.63^{\mathrm{a}}$ & $>100^{\mathrm{a}}$ \\
$\mathbf{1 0}$ & $40.56 \pm 1.41^{\mathrm{a}}$ & $>100^{\mathrm{a}}$ \\
$\mathbf{1 1}$ & $>100^{\mathrm{c}}$ & $>100^{\mathrm{a}}$ \\
$\mathbf{1 2}$ & $>100^{\mathrm{c}}$ & $>100^{\mathrm{a}}$ \\
$\mathbf{1 3}$ & $>100^{\mathrm{c}}$ & $>100^{\mathrm{a}}$ \\
$\mathbf{1 4}$ & $>100^{\mathrm{c}}$ & $>100^{\mathrm{a}}$ \\
$\mathbf{1 5}$ & $>100^{\mathrm{c}}$ & $>100^{\mathrm{a}}$ \\
\hline
\end{tabular}

Values represent means \pm S.D. of three determinations. Values bearing different superscripts in the same column are significantly different $(p<0.05)$.

\section{Experimental}

\subsection{General Methods}

Sephadex LH-20 column (10-25 $\mu \mathrm{m}$, GE Healthcare Bio-Science AB, Uppsala, Sweden) and Daisogel ODS-B with MPLC system $(5 \times 80 \mathrm{~cm}$, Mitsubishi Chemical Co., Tokyo, Japan), 110UV/VIS detector (Gilson, Middleton, WI, USA) and TBP 5002 pump (Tauto Biotech, Shanghai, China) were used for column chromatography. TLC was carried out on pre-coated silica gel $60 \mathrm{~F}_{254}$ plates (Merck, Darmstadt, Germany); spots were detected under UV radiation (254 $\mathrm{nm}$ ) and by spraying with $\mathrm{FeCl}_{3}$ solution and $10 \% \mathrm{H}_{2} \mathrm{SO}_{4}$ followed by heating. The ${ }^{1} \mathrm{H}$ - and ${ }^{13} \mathrm{C}-\mathrm{NMR}$ spectra were recorded at $300 \mathrm{MHz}$ on a Gemini 2000 instrument (Varian, Palo Alto, CA, USA) and ${ }^{1} \mathrm{H}-\mathrm{NMR}, 600 \mathrm{MHz} ;{ }^{13} \mathrm{C}-\mathrm{NMR}$, $150 \mathrm{MHz}$ on a Varian VNS (Varian, Palo Alto, CA, USA) and the resolution fast atom bombardment mass spectrum (LRFAB-MS) were measured with a JMSAX505WA instrument (JEOL, Tokyo, Japan).

\subsection{Plant Material}

The leaves of CL (1.8 kg) were collected from the Korea Forest Research Institute, Suwon, Korea in September 2010 and certified by Minwon Lee (Phamacognosy Lab, College of Pharmacy, Chung-Ang University). The voucher specimen (CL2010-9) was deposited at the herbarium of the College of Pharmacy, Chung-Ang University.

\subsection{Extraction and Isolation}

The dry leaves of CL (1.8 kg) were extracted for $72 \mathrm{~h}$ at room temperature with $80 \%$ aqueous $\mathrm{MeOH}(8.0 \mathrm{~L})$. The concentrated extracts were subjected to preparative thin layer 
chromatography (TLC) using as solvent systems C:M:W (chloroform:MeOH: $\mathrm{H}_{2} \mathrm{O}=6: 4: 1$ ) or B:E:F (benzene:ethyl formate:formic acid $=1: 7: 1)$. The spots were detected under UV radiation $(254 \mathrm{~nm})$ and by spraying with $\mathrm{FeCl}_{3}$ and $10 \% \mathrm{H}_{2} \mathrm{SO}_{4}$ or anisaldehyde- $\mathrm{H}_{2} \mathrm{SO}_{4}$ followed by heating. The CL aqueous layer $(270 \mathrm{~g})$ was concentrated and applied to a Sephadex LH-20 column (10-25 $\mu \mathrm{m}, 2 \mathrm{~kg}$, $10 \times 80 \mathrm{~cm}$ ), and eluted with $\mathrm{H}_{2} \mathrm{O}$ containing increasing proportions of $\mathrm{MeOH}$ to afford from sub-fraction, 1 (134.6 g), 2 (23.5 g), 3 (10.9 g), 4 (33.3 g) and 5 (49.6 g). Repeated column chromatography of sub-fraction 2 on Daisogel ODS-B with MPLC system $(300 \mu \mathrm{m}, 500 \mathrm{~g}, 5 \times 80 \mathrm{~cm}$, Mitsubishi Chemical Co.), using a $\mathrm{H}_{2} \mathrm{O}: \mathrm{MeOH}$ gradient yielded compounds 2 (481 mg), 4 (300 mg), 5 $(15.5 \mathrm{~g}), \mathbf{6}(87.5 \mathrm{mg})$. The sub-fraction 3 gave compounds 1 (370 mg), 3 (692 $\mathrm{mg})$, sub-fraction 4 afforded compounds 7 (810 mg), 8 (4.2 g), $11(1.1 \mathrm{~g}), \mathbf{1 2}(10.3 \mathrm{~g}), \mathbf{1 3}(126.3 \mathrm{mg}), \mathbf{1 4}(1.8 \mathrm{~g})$ and 15 (475 mg). Finally, sub-fraction 5 contained compound $\mathbf{9}(458 \mathrm{mg})$ and $\mathbf{1 0}(11 \mathrm{~g})$, respectively (Figure 1).

3-Caffeoyl quinic acid Methyl ester (1). Dark brown amorphous powder; Negative FAB-MS m/z: 367 $[\mathrm{M}-\mathrm{H}]^{-}, 179$ [M-quinic acid methyl ester:caffeic acid] ${ }^{-}{ }^{1} \mathrm{H}-\mathrm{NMR}\left(300 \mathrm{MHz}, \mathrm{MeOH}-d_{4}\right): \delta 2.17(2 \mathrm{H}$, m, H-6'), 2.22 (2H, m, H-2'), $3.75\left(3 \mathrm{H}, \mathrm{s}, \mathrm{OCH}_{3}\right), 3.77$ (1H, m, H-4'), 4.19 (1H, m, H-5'), 5.33 (1H, m, H-3'), $6.29(1 \mathrm{H}, \mathrm{d}, J=16.2 \mathrm{~Hz}, \mathrm{H}-8), 6.90(1 \mathrm{H}, \mathrm{d}, J=8.4 \mathrm{~Hz}, \mathrm{H}-5), 7.06(1 \mathrm{H}, \mathrm{dd}, J=0.6,8.4 \mathrm{~Hz}$, H-6), $7.18(1 \mathrm{H}, \mathrm{d}, J=0.6 \mathrm{~Hz}, \mathrm{H}-2), 7.57$ (1H, d, $J=16.2 \mathrm{~Hz}, \mathrm{H}-7)$.

4-Caffeoyl quinic acid (2). Dark brown amorphous powder; Negative FAB-MS m/z: 353 [M-H], 179 [M-quinic acid:Caffeic acid] ${ }^{-}{ }^{1} \mathrm{H}-\mathrm{NMR}\left(300 \mathrm{MHz}, \mathrm{MeOH}-d_{4}\right): \delta 1.90\left(2 \mathrm{H}, \mathrm{m}, \mathrm{H}-2^{\prime}\right), 2.06$ (2H, m, H-6'), $3.73\left(1 \mathrm{H}, \mathrm{m}, \mathrm{H}-5^{\prime}\right), 4.13\left(1 \mathrm{H}, \mathrm{m}, \mathrm{H}-3^{\prime}\right), 5.18\left(1 \mathrm{H}, \mathrm{m}, \mathrm{H}-4^{\prime}\right), 6.26(1 \mathrm{H}, \mathrm{d}, J=16.8 \mathrm{~Hz}, \mathrm{H}-8), 6.76(1 \mathrm{H}, \mathrm{d}$, $J=7.8 \mathrm{~Hz}, \mathrm{H}-5), 7.00(1 \mathrm{H}, \mathrm{dd}, J=1.8,7.8 \mathrm{~Hz}, \mathrm{H}-6), 7.04(1 \mathrm{H}, \mathrm{s}, \mathrm{H}-2), 7.48$ (1H, d, $J=16.8 \mathrm{~Hz}, \mathrm{H}-7)$.

3-Caffeoyl quinic acid (3). Dark brown amorphous powder; ${ }^{1} \mathrm{H}-\mathrm{NMR}\left(300 \mathrm{MHz}, \mathrm{MeOH}-d_{4}\right): \delta 2.20$ (2H, m, H-6'), 2.24 (2H, m, H-2'), 3.77 (1H, m, H-4'), 4.22 (1H, m, H-5'), 5.37 (1H, m, H-3'), 6.29 (1H, d, $J=15.6 \mathrm{~Hz}, \mathrm{H}-8), 6.87(1 \mathrm{H}, \mathrm{d}, J=8.1 \mathrm{~Hz}, \mathrm{H}-5), 7.13(1 \mathrm{H}, \mathrm{dd}, J=1.8,8.1 \mathrm{~Hz}, \mathrm{H}-6), 7.15(1 \mathrm{H}, \mathrm{d}$, $J=1.8 \mathrm{~Hz}, \mathrm{H}-2), 7.57(1 \mathrm{H}, d, J=15.6 \mathrm{~Hz}, \mathrm{H}-7)$.

3-O-galloyl- $\beta$-D-glucopyranoside (4). Yellow amorphous powder; Negative FAB-MS $m / z$ : 331 $[\mathrm{M}-\mathrm{H}]^{-}, 169$ [M-glucoside:Gallic aicd] ${ }^{-},{ }^{1} \mathrm{H}-\mathrm{NMR}\left(300 \mathrm{MHz}, \mathrm{MeOH}-d_{4}\right): \delta 3.20\left(1 \mathrm{H}, \mathrm{m}, \mathrm{H}-3^{\prime}\right), 3.33$ (1H, m, H-4'), 3.54 (2H, m, H-6', 5'), 3.76 (1H, m, H-6'), $3.81\left(1 \mathrm{H}, \mathrm{m}, \mathrm{H}-2^{\prime}\right), 5.57$ (1H, d, J = 7.8Hz, H-1'), 7.07 (2H, s, H-2, 6), ${ }^{13} \mathrm{C}-\mathrm{NMR}\left(150 \mathrm{MHz}, \mathrm{MeOH}-d_{4}\right): \delta 60.8\left(\mathrm{C}-6^{\prime}\right), 69.8\left(\mathrm{C}-4^{\prime}\right), 73.2\left(\mathrm{C}-2^{\prime}\right)$, 76.8 (C-3'), 78.5 (C-5'), 94.9 (C-1'), 109.3 (C-2. 6), 119.3 (C-1), 139.0 (C-4), 145.8 (C-3, 5), 165.0 (C-7).

Bergenin (5). White amorphous powder; Negative FAB-MS m/z: $327[\mathrm{M}-\mathrm{H}]^{-},{ }^{1} \mathrm{H}-\mathrm{NMR}(600 \mathrm{MHz}$, $\left.\mathrm{MeOH}-d_{4}\right): \delta 3.47(1 \mathrm{H}, \mathrm{t}, 1 \mathrm{H} J=9.3 \mathrm{~Hz}, \mathrm{H}-3$ '), $3.73(2 \mathrm{H}, \mathrm{m}, \mathrm{H}-11), 3.84(1 \mathrm{H}, \mathrm{t}, 1 \mathrm{H}, \mathrm{t}, J=8.1 \mathrm{~Hz}$, H-2'), $3.89\left(3 \mathrm{H}, \mathrm{s}, \mathrm{OCH}_{3}\right), 4.04\left(1 \mathrm{H}, \mathrm{t}, J=9.6 \mathrm{~Hz}, \mathrm{H}-4{ }^{\prime} \beta\right), 4.09\left(1 \mathrm{H}, \mathrm{t}, J=9.6 \mathrm{~Hz}, \mathrm{H}-4^{\prime} \alpha\right), 4.96(1 \mathrm{H}, \mathrm{d}$, $\left.J=10.2 \mathrm{~Hz}, \mathrm{H}-1^{\prime}\right), 7.07$ (1H, s, H-7), ${ }^{13} \mathrm{C}-\mathrm{NMR}\left(150 \mathrm{MHz}, \mathrm{MeOH}-d_{4}\right): \delta 59.4(\mathrm{C}-12), 61.2(\mathrm{C}-11)$,

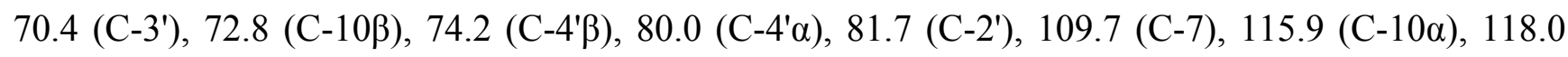
(C-6 $\alpha), 140.8$ (C-9), 148.0 (C-10), 150.8 (C-8), 164.3 (C-6).

Norbergenin (6). White amorphous powder; ${ }^{1} \mathrm{H}-\mathrm{NMR}\left(300 \mathrm{MHz}, \mathrm{MeOH}-d_{4}\right): \delta 3.47(1 \mathrm{H}, \mathrm{t}, 1 \mathrm{H} J=9.0 \mathrm{~Hz}$, H-3'), 3.73 (2H, m, H-11), 3.84 (1H, t, $\left.J=8.1 \mathrm{~Hz}, \mathrm{H}-2^{\prime}\right), 4.04$ (1H, t, $\left.J=9.6 \mathrm{~Hz}, \mathrm{H}-4{ }^{\prime} \beta\right), 4.09$ (1H, d, $\left.J=9.6 \mathrm{~Hz}, \mathrm{H}-4^{\prime} \alpha\right), 5.26(1 \mathrm{H}, \mathrm{d}, J=10.2 \mathrm{~Hz}$, anomeric H-1'), $7.08(1 \mathrm{H}, \mathrm{s}, \mathrm{H}-7)$. 
11-Galloylbergenin (7). White amorphous powder; Negative FAB-MS m/z: $479[\mathrm{M}-\mathrm{H}]^{-} .{ }^{1} \mathrm{H}-\mathrm{NMR}$ $\left(300 \mathrm{MHz}, \mathrm{MeOH}-d_{4}\right): \delta 3.70\left(1 \mathrm{H}, \mathrm{t}, J=9.3, \mathrm{H}-3\right.$ '), $3.88\left(3 \mathrm{H}, \mathrm{s}, \mathrm{OCH}_{3}\right), 3.95(2 \mathrm{H}, \mathrm{m}, \mathrm{H}-11), 4.13(1 \mathrm{H}$, t, $\left.J=10.2 \mathrm{~Hz}, \mathrm{H}-2^{\prime}\right), 4.30\left(1 \mathrm{H}, \mathrm{dd}, J=3,12 \mathrm{~Hz}, \mathrm{H}-4{ }^{\prime} \beta\right), 4.66\left(1 \mathrm{H}, \mathrm{dd}, J=12,6,6 \mathrm{~Hz}, \mathrm{H}-4^{\prime} \alpha\right), 5.05(1 \mathrm{H}$, $\left.\mathrm{d}, J=10.2 \mathrm{~Hz}, \mathrm{H}-1^{\prime}\right), 7.11(1 \mathrm{H}, \mathrm{s}, \mathrm{H}-7), 7.18(1 \mathrm{H}, \mathrm{s}, \mathrm{H}-2,6),{ }^{13} \mathrm{C}-\mathrm{NMR}\left(150 \mathrm{MHz}, \mathrm{MeOH}-d_{4}\right): \delta 60.3$

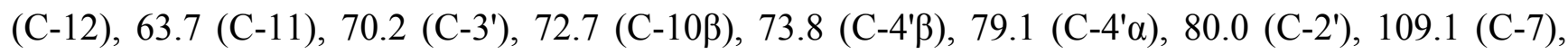

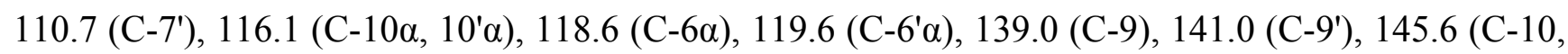
10'), 148.2 (C-8), 151.0 (C-8'), 166.3 (C-6), 166.8 (C-6').

Tellimagrandin I (8). Dark yellow amorphous powder; Positive FAB-MS m/z: $787[\mathrm{M}+\mathrm{H}]^{+},{ }^{1} \mathrm{H}-\mathrm{NMR}$ $\left(600 \mathrm{MHz}\right.$, Aceton- $\left.d_{4}+\mathrm{D}_{2} \mathrm{O}\right): \delta 3.79(1 \mathrm{H}, \mathrm{dd}, J=13.2,1.8 \mathrm{~Hz}, \mathrm{H}-6 " \beta), 3.87(1 \mathrm{H}, \mathrm{dd}, J=13.2,1.8 \mathrm{~Hz}$, H-6" $\alpha), 4.28(1 \mathrm{H}, \mathrm{dd}, J=6.6,9.6 \mathrm{~Hz}, \mathrm{H}-4 " \alpha), 4.69(1 \mathrm{H}, \mathrm{dd}, J=6.6,9.6 \mathrm{~Hz}, \mathrm{H}-4 " \beta), 5.11(1 \mathrm{H}, \mathrm{m}$,

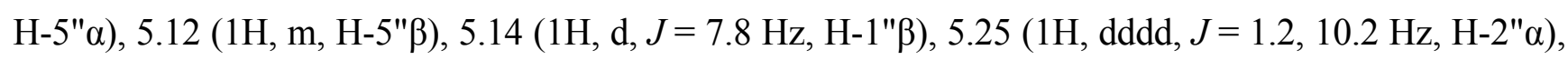
$5.27(1 \mathrm{H}, \mathrm{dddd}, J=1.2,10.2 \mathrm{~Hz}, \mathrm{H}-2 " \beta), 5.29(1 \mathrm{H}, \mathrm{t}, J=6.6 \mathrm{~Hz}, \mathrm{H}-6 " \beta), 5.31(1 \mathrm{H}, \mathrm{t}, J=6.6 \mathrm{~Hz}$, H-6" $\alpha), 5.57$ (1H, d, $J=3.6 \mathrm{~Hz}, \mathrm{H}-1 " \alpha), 5.62(1 \mathrm{H}, \mathrm{t}, J=10.2,9.6 \mathrm{~Hz}, \mathrm{H}-3 " \beta), 5.89$ (1H, t, $J=10.2$, $9.6 \mathrm{~Hz}, \mathrm{H}-3 " \alpha), 6.49$ (2H, s, HHDP-3'), 6.66 (2H, s, HHDP-3'), 6.95 (2H, s, G-2, 6), 6.99 (2H, s, G-2, 6), $7.05(2 \mathrm{H}, \mathrm{s}, \mathrm{G}-2,6), 7.06(2 \mathrm{H}, \mathrm{s}, \mathrm{G}-2,6),{ }^{13} \mathrm{C}-\mathrm{NMR}\left(150 \mathrm{MHz}\right.$, Aceton- $\left.d_{4}+\mathrm{D}_{2} \mathrm{O}\right): \delta 63.0(\mathrm{C}-6 " \alpha$,

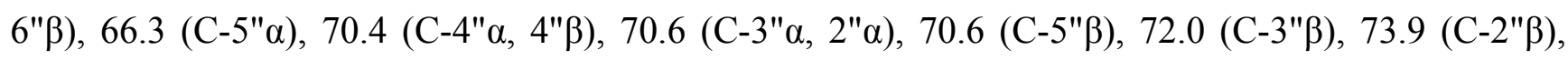

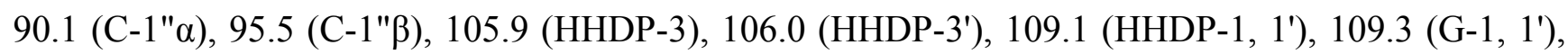
115.6 (G-2), 118.8 (G-2'), 124.3 (HHDP-5, 5'), 124.9 (HHDP-2, 2'), 135.6 (G-4'), 138.9 (G-4), 144.6 (HHDP-4, 4'), 144.8 (HHDP-6, 6'), 145.4 (G-3), 145.7 (G-3'), 165.5 (-COO ), 165.8 (-COO), 167.3 (-COO), 168.0 (-COO).

Tellimagrandin II (9). Dark yellow amorphous powder; Negative FAB-MS m/z: 953 [M-H] $]^{-}, 169$ [M-tellimagrandin I:Gallic acid] ${ }^{-}{ }^{1} \mathrm{H}-\mathrm{NMR}\left(600 \mathrm{MHz}\right.$, Aceton- $\left.d_{4}+\mathrm{D}_{2} \mathrm{O}\right): \delta 3.92(1 \mathrm{H}, \mathrm{d}, J=6.6 \mathrm{~Hz}$, H-6"ß) 4.58 (1H, dd, $J=1.2,6.6 \mathrm{~Hz}, \mathrm{H}-4 "), 5.26$ (1H, t, $J=8.4,9.6 \mathrm{~Hz}, \mathrm{H}-5 "), 5.38$ (1H, dd, $J=6.6 \mathrm{~Hz}$, H-6" $\alpha), 5.64(1 \mathrm{H}, \mathrm{dd}, J=1.2,8.4 \mathrm{~Hz}, \mathrm{H}-2 "), 5.87(1 \mathrm{H}, \mathrm{t}, J=8.4,9.6 \mathrm{~Hz}, \mathrm{H}-3 "), 6.22(1 \mathrm{H}, \mathrm{d}, J=8.4$ Hz, H-1"), 6.68 (2H, s, HHDP-3'), 7.00 (2H, s, G-2, 6), 7.03 (2H, s, G-2, 6), 7.13 (2H, s, G-2, 6), ${ }^{13} \mathrm{C}-\mathrm{NMR}\left(150 \mathrm{MHz}\right.$, Aceton- $\left.d_{4}+\mathrm{D}_{2} \mathrm{O}\right): \delta 62.2$ (C-6"), 69.8 (C-4"), 71.0 (C-2"), 72.2 (C-5"), 72.4 (C-3"), 92.8 (C-1"), 107.0 (HHDP-3), 107.2 (HHDP-3'), 109.2 (HHDP-1, 1'), 109.3 (G-1', 1",1"'), 114.8 (G-2"'), 118.8 (G-2', 2"), 124.8 (HHDP-2, 2'), 125.0 (HHDP-5, 5'), 135.6 (G-4", 4"'), 138.9 (G-4'), 144.6 (HHDP-4, 4'), 144.9 (HHDP-6, 6'), 145.1 (G-3", 3"'), 145.3 (G-3'), 164.2 (-COO), 165.0 (-COO), 165.6 (-COO), 166.9 (-COO), 167.3 (-COO).

Casuarinin (10). Dark yellow amorphous powder; Positive FAB-MS $m / z$ : $937[\mathrm{M}+\mathrm{H}]^{+},{ }^{1} \mathrm{H}-\mathrm{NMR}$ $\left(600 \mathrm{MHz}\right.$, Aceton- $\left.d_{4}+\mathrm{D}_{2} \mathrm{O}\right): \delta 3.72(1 \mathrm{H}, \mathrm{t}, J=13.2 \mathrm{~Hz}, \mathrm{H}-6 " \beta), 3.79(1 \mathrm{H}, \mathrm{t}, J=13.2 \mathrm{~Hz}, \mathrm{H}-6 " \alpha), 4.43$ $(1 \mathrm{H}, \mathrm{dd}, J=6.6,9.6 \mathrm{~Hz}, \mathrm{H}-4 " \alpha), 4.46(1 \mathrm{H}, \mathrm{dd}, J=6.6,9.6 \mathrm{~Hz}, \mathrm{H}-4 " \beta), 4.83$ (1H, m, H-5" $\alpha), 4.88$ (1H, m,

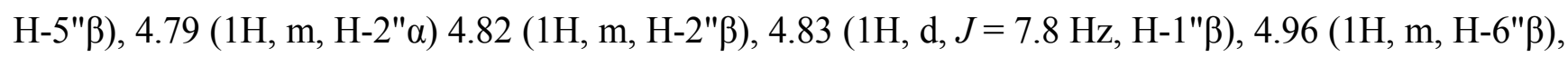
$4.98(1 \mathrm{H}, \mathrm{m}, \mathrm{H}-6 " \alpha), 5.32(1 \mathrm{H}, \mathrm{d}, J=3.6 \mathrm{~Hz}, \mathrm{H}-1 " \alpha), 5.47$ (1H, t, $J=10.2,9.6 \mathrm{~Hz}, \mathrm{H}-3 " \beta), 5.66(1 \mathrm{H}$, t, $J=10.2,9.6 \mathrm{~Hz}, \mathrm{H}-3 " \alpha), 5.90$ (2H, s, G-2, 6), 6.26 (2H, s, HHDP-3), 6.81 (2H, s, HHDP-3), 6.86 (2H, s, HHDP-3), 6.92 (2H, s, HHDP-3), ${ }^{13} \mathrm{C}-\mathrm{NMR}\left(150 \mathrm{MHz}\right.$, Aceton- $\left.d_{4}+\mathrm{D}_{2} \mathrm{O}\right): \delta 63.0(\mathrm{C}-6 " \alpha, 6 " \beta)$,

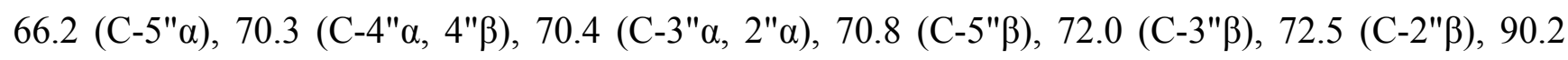

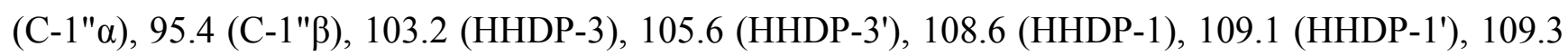


(G-1, 1'), 115.5 (G-2), 118.8 (G-2'), 124.0 (HHDP-5, 5'), 124.2 (HHDP-2, 2'), 135.5 (G-4'), 139.0 (G-4), 142.8 (HHDP-4, 4'), 144.7 (HHDP-6, 6'), 145.5 (G-3), 145.7 (G-3'), 165.6 (-COO), 165.8 (-COO), 167.3 (-COO), 168.0 (-COO).

Quercetin (11). Yellow amorphous powder; ${ }^{1} \mathrm{H}-\mathrm{NMR}\left(300 \mathrm{MHz}, \mathrm{DMSO}-d_{\sigma}+\mathrm{D}_{2} \mathrm{O}\right): \delta 6.19(1 \mathrm{H}, \mathrm{d}$, $\left.J=2.1 \mathrm{~Hz}, \mathrm{H}-6), 6.41(1 \mathrm{H}, \mathrm{d}, J=1.8 \mathrm{~Hz}, \mathrm{H}-8), 6.89(1 \mathrm{H}, \mathrm{d}, J=8.4 \mathrm{~Hz}, \mathrm{H}-5)^{\prime}\right), 7.55$ (1H, dd, $J=2.1$, $\left.8.4 \mathrm{~Hz}, \mathrm{H}-6^{\prime}\right), 7.68$ (1H, d, $\left.J=2.1 \mathrm{~Hz}, \mathrm{H}-2^{\prime}\right)$.

Quercitrin (12). Yellow amorphous powder; Negative FAB-MS $m / z$ : $447[\mathrm{M}-\mathrm{H}]^{-}, \quad 301$ [M-rhamnose:Quercetin] ${ }^{-},{ }^{1} \mathrm{H}-\mathrm{NMR}\left(300 \mathrm{MHz}, \mathrm{DMSO}-d_{\sigma}+\mathrm{D}_{2} \mathrm{O}\right): \delta 0.80(3 \mathrm{H}, \mathrm{d}, J=6.6 \mathrm{~Hz}, \mathrm{H}-6 ")$, 3.18 (3H, m, H-3", 5", 4"), 3.97 (1H, dd, J=4.5 Hz, H-2"), 5.24 (1H, s, H-1"), 6.22 (1H ,d, $J=1.8 \mathrm{~Hz}$, H-6), 6.41 (1H, d, $J=1.8 \mathrm{~Hz}, \mathrm{H}-8), 6.88\left(1 \mathrm{H}, \mathrm{d}, J=8.4 \mathrm{~Hz}, \mathrm{H}-5^{\prime}\right), 7.26$ (1H, dd, $\left.J=2.1,8.4 \mathrm{~Hz}, \mathrm{H}-6^{\prime}\right)$, $7.29\left(1 \mathrm{H}, \mathrm{d}, J=2.1 \mathrm{~Hz}, \mathrm{H}-2^{\prime}\right)$.

Quercetin 3-O- $\beta$-D-glucuronide (13). Yellow amorphous powder; Negative FAB-MS m/z: 477 [M-H] , 301 [M-glucuronide:Quercetin] ${ }^{-}{ }^{1} \mathrm{H}-\mathrm{NMR}\left(300 \mathrm{MHz}, \mathrm{DMSO}-d_{\sigma}+\mathrm{D}_{2} \mathrm{O}\right): \delta 3.08(2 \mathrm{H}, \mathrm{m}, \mathrm{H}-6 "), 3.21$ (1H, m, H-2"), 3.33 (2H, m ,H-4", 3"), 3.58 (1H, m, H-5"), 5.48 (1H, d, J= 7.8 Hz, H-1"), 6.22 (1H, d, $J=2.1 \mathrm{~Hz}, \mathrm{H}-6), 6.42(1 \mathrm{H}, \mathrm{d}, J=2.1 \mathrm{~Hz}, \mathrm{H}-8), 6.87\left(1 \mathrm{H}, \mathrm{d}, J=8.7 \mathrm{~Hz}, \mathrm{H}-5{ }^{\prime}\right), 7.57(1 \mathrm{H}, \mathrm{dd}, J=2.1$, $\left.8.7 \mathrm{~Hz}, \mathrm{H}-6^{\prime}\right), 7.59\left(1 \mathrm{H}, \mathrm{d}, J=2.1 \mathrm{~Hz}, \mathrm{H}-2^{\prime}\right)$.

Datiscetin 3-O- $\beta$-D-rhamnpyranoside (14). Yellow amorphous powder; Positive FAB-MS m/z: 447 $[\mathrm{M}-\mathrm{H}]^{-}, 283$ [M-rhamnose:Datiscetin] ${ }^{-}{ }^{1} \mathrm{H}-\mathrm{NMR}\left(300 \mathrm{MHz}, \mathrm{DMSO}-d_{6}+\mathrm{D}_{2} \mathrm{O}\right): \delta 0.83(3 \mathrm{H}, \mathrm{d}, J=6.6 \mathrm{~Hz}$, H-6"), 3.18 (3H, m, H-3", 5", 4"), 3.96 (1H, dd, $J=4.5$ Hz, H-2"), 5.24 (1H, s, H-1"), 6.22 (1H, d, $J=1.2 \mathrm{~Hz}, \mathrm{H}-6), 6.42(1 \mathrm{H}, \mathrm{d}, J=1.2 \mathrm{~Hz}, \mathrm{H}-8), 6.88(2 \mathrm{H}, \mathrm{d}, J=7.6,8.4 \mathrm{~Hz}, \mathrm{H}-3$ ', 5'), 7.29 (1H, dd, $J=7.6,8.4 \mathrm{~Hz}, \mathrm{H}-4$ '), $7.59\left(1 \mathrm{H}, \mathrm{d}, J=7.5 \mathrm{~Hz}, \mathrm{H}-6^{\prime}\right)$.

Myricetin 3-O- $\beta$-D-rhamnopyranoside (15). Yellow amorphous powder; Negative FAB-MS m/z: 447 $[\mathrm{M}-\mathrm{H}]^{-}, 317$ [M-rhamnose:Myricetin] ${ }^{-}{ }^{1} \mathrm{H}-\mathrm{NMR}\left(300 \mathrm{MHz}, \mathrm{DMSO}-d_{6}+\mathrm{D}_{2} \mathrm{O}\right): \delta 0.81(3 \mathrm{H}, \mathrm{d}, J=6.6 \mathrm{~Hz}$, H-6"), 3.15 (1H, d, $J=9.4, \mathrm{H}-5 "), 3.16$ (1H, t, $J=9.4,6.1, \mathrm{H}-, 4 "), 3.32$ (1H, dd, $J=9.4,3.3 \mathrm{~Hz}, \mathrm{H}-3 ")$, 3.97 (1H, bs, $J=1.4$ Hz, H-2"), 5.19 (1H, s, H-1"), 6.39 (2H, d, J=1.2 Hz, H-6, 8), 6.88 (2H, s, H-2', 6').

\subsection{Antioxidant Activity}

\subsubsection{Measurement of DPPH Radical Scavenging Activity}

Each sample in absolute EtOH was added to DPPH solution $(0.1 \mathrm{mM}$, in absolute EtOH). After mixing gently for $30 \mathrm{~min}$, optical densities were measured at $518 \mathrm{~nm}$ using a microplate reader (TECAN, Salzburg, Austria). L-Ascorbic acid (Sigma, St. Louis, MO, USA) was used as a positive control [21].

\subsubsection{Xanthine Oxidase Superoxide Scavenging Activity}

After each sample was added to $50 \mathrm{mM}$ phosphate buffer $(\mathrm{pH} 7.5)$ containing $0.05 \mathrm{mM}$ EDTA, $0.2 \mathrm{mM}$ hypoxanthine and $0.1 \mathrm{mM} \mathrm{NBT}$, xanthine oxidase $(1.2 \mathrm{U} / \mu \mathrm{L})$ was then added to this mixture. 
After mixing gently for $10 \mathrm{~min}$, optical densities were measured at $612 \mathrm{~nm}$ using a microplate reader (Tecan). Allopurinol was used as a positive control [22].

\subsection{Anti-Proliferation and Cytotoxicity Assays}

\subsubsection{Cell Culture}

RAW 264.7 macrophage, DU-145 and LNCaP human prostate cancer cell lines were purchased from the Korean Cell Line Bank. The RAW 264.7 macrophage cell and human prostate cancer cell lines was grown at $37{ }^{\circ} \mathrm{C}$ in a humidified atmosphere $\left(5 \% \mathrm{CO}_{2}\right)$ in DMEM (Sigma) and RPMI (Sigma) containing $10 \%$ fetal bovine serum, $10 \mathrm{IU} / \mathrm{mL}$ penicillin $\mathrm{G}$ and $100 \mu \mathrm{g} / \mathrm{mL}$ streptomycin (Gibco BRL, Grand Island, NY, USA).

\subsubsection{Measurement of Cell Viability}

After culturing of RAW 264.7 macrophage $\left(3.5 \times 10^{5}\right.$ cells $/ 200 \mu \mathrm{L}$ medium $)$ in 96-well plates and incubating for $2 \mathrm{~h}$, in 96-well plates and incubating for $24 \mathrm{~h}$, the cells were treated with the test samples. The cells were incubated for an additional $24 \mathrm{~h}$, and the medium was replaced with fresh medium containing $0.5 \mathrm{mg} / \mathrm{mL}$ 3-(4,5-dimethylthiazol-2-yl)-2,5-diphenyltetrazolium bromide (MTT) (Sigma). Incubation was continued for $4 \mathrm{~h}$ at $37{ }^{\circ} \mathrm{C}$. The medium was then removed and the MTT-formazan produced was dissolved in dimethyl sulfoxide (DMSO). The extent of the reduction of MTT to dark purple crystals within the cells was quantified by measuring the absorbance at $540 \mathrm{~nm}$ using the microplate reader (Tecan) [24].

\subsubsection{Anti-Proliferation Assays}

After culturing of DU145 and LNCaP human prostate cancer cell lines $\left(1 \times 10^{4}\right.$ cells/ $200 \mu \mathrm{L}$ medium) in 24-well plates and incubating for $24 \mathrm{~h}$, the cells were treated with the test samples. Plates were incubated for $72 \mathrm{~h}$ after which bioassays were performed. LNCaP cells were handled in a similar manner, and the medium was replaced with fresh medium containing $0.5 \mathrm{mg} / \mathrm{mL} 3-(4,5$-dimethylthiazol2-yl)-2,5-diphenyltetrazolium bromide (MTT) (Sigma). Incubation was continued for $4 \mathrm{~h}$ at $37{ }^{\circ} \mathrm{C}$. The medium was then removed and the MTT-formazan produced was dissolved in dimethyl sulfoxide (DMSO). The extent of the reduction of MTT to dark purple crystals within the cells was quantified by measuring the absorbance at $540 \mathrm{~nm}$ using the microplate reader (Tecan) [24].

\subsection{Statistical Analysis}

All data are expressed as mean \pm S.D. Values were performed by one-way analysis of variance (ANOVA) followed by Student-Newman-Keuls (S-N-K) test using the SPSS software package; the values were considered significantly different when the $p$ value was less than 0.05 .

\section{Conclusions}

Fifteen phenolic compounds 1-15, inclusing three caffeoyl derivatives 1-3, four gallotannins 4-7, three ellagitannins 8-10 and five flavonoids 11-15 were isolated from CL. DPPH radical scavenging 
and superoxide scavenging activity, as well as, anti-proliferative activity on prostate cancer call lines (DU145 and LNCaP) of the isolated compounds were evaluated. As a results, the ellagitannin derivatives 8-10 showed potent anti-oxidative and anti-proliferative activities on prostate cancer cell lines. These results suggested that CL extract and its phenolic compounds could potentially be developed as ingredients with anti-oxidative and androgen sensitive anti-proliferation activity.

\section{Acknowledgements}

This study was supported by Basic Science Research Program through the National Research Foundation of Korea (NRF) funded by the Ministry of Education, Science and Technology (2010-0022929).

\section{References}

1. Zhang, Z.; Zhang, H.; Peter, K.E. Hamaelidaceae. In Flora of China; Science Press \& Missouri Botanical Garden: Beijing, China and St. Louis, MO, USA, 2003; Volume 9, pp. 18-42.

2. Huafu, W.; Gordon, J.P.; Keith, H. Determination of hamameli-tannin, catechins and gallic acid in witch hazel bark, twig and leaf by HPLC. J. Pharm. Biomed. Anal. 2003, 33, 539-544.

3. Korting, H.C.; Schäfer-Korting, M.; Klövekorn, W.; Klövekorn, G.; Martin, C.; Laux, P. Comparative efficacy of hamamelis distillate and hydrocortisone cream in atopic eczema. Eur. J. Clin. Pharmacol. 1995, 48, 461-465.

4. Dauer, A.; Metzner, P.; Schimmer, O. Proanthocyanidins from the bark of Hamamelis virginiana exhibit antimutagenic properties against nitroaromatic compounds. Planta Med. 1998, 64, 324-327.

5. Dauer, A.; Rimpler, H.; Hensel, A. Polymeric proanthocyanidins from the bark of Hamamelis virginiana L. Planta Med. 2003, 69, 89-91.

6. Hideo, I.; Hideko, M.; Ideko, M.; Noriko, O.; Ryo, K. 3-O-feruloyl-4-O-caffeoylquinic acid from coffee beans. Phytochemistry 1985, 24, 630-632.

7. Akdemir, Z.S.; Tatli, I.I.; Saracoglu, I.; Ismailoglu, U.B.; Sahin-Erdemli, I.; Caliş, I. Polyphenolic compounds from Geranium prat-ense and their free radical scavenging activities. Phytochemistry 2001, 56, 189-193.

8. Zamarrud, A.I.; Hussain; H; Ahmad, V.U.; Qaiser; M; Amyn; A; Mohammad, F.V. Two new antioxidant Bergen-in derivatives from the stem of Rivea hypocrateriformis. Fitoterapia 2011, 82, $722-725$.

9. Dong, W.; Zhu, H.T.; Zhang, Y.J.; Yang, C.R. A carbon-carbon-coupled dimeric bergenin derivative biotransformed by Pleu-rotus ostreatus. Bioorg. Med. Chem. Lett. 2005, 15, 4073-4075.

10. Lee, M.W.; Tanaka, T.; Nonaka, G.I.; Nishioka, I. Hirsunin, an ellagitannin with a diarylheptanoid moiety, from Alnus hirsuta var. Microphylla. Phytochemistry 1992, 31, 967-970.

11. Jin, Z.X.; Ito, H.; Yoshida, T. Dimeric and trimeric ellagitannins from corylus heterophylla. Phytochemistry 1998, 48, 333-338.

12. Jeng, D.S.; Toshihiko, O.; Shunro, K.; Mitsuo, N. Tannin antioxidants from Osbeckia chinensis. Phytochemistry 1988, 27, 1315-1319.

13. Lommen, A.; Godejohann, M.; Venema, D.P.; Hollman, P.C.H.; Spraul, M. Application of directly coupled HPLC-NMR-MS to the identification and confirmation of quercetin glycosides and phloretin glycosides in apple peel. Anal. Chem. 2000, 72, 1793-1797. 
14. Philippe, O.D.B.; Dijoux, M.G.; Gilbert, C.; Anne-marie, M.I. Quercitrin T-sulphate fromleaves of leea G uinensis. Phytochemistry 1998, 47, 1171-1173.

15. Mohamed, B.; Aziz, A.; Christian, R. Regio- and ster-eoselective synthesis of the major metabolite of quercetin, quercetin-3-O- $\beta$-D-glucuronide. Tetrahedron Lett. 2002, 43, 6263-6266.

16. Lee, S.H.; Park, Y.H.; Moon, B.H.; Lee, E.J.; Hong, S.W.; Lim, Y.H. Substitution effect of hydroxyl groups on the ${ }^{1} \mathrm{H}$ and ${ }^{13} \mathrm{C}$ Chemical Shifts in Hydroxyflavonols. Bull. Korean Chem. Soc. 2008, $29,1597-1600$.

17. Lee, J.I.; Kong, C.S.; Jung, M.E.; Hong, J.W.; Noh, I.; Seo, Y.W. Peroxynitrite-scavenging activity of the halophyte limon-ium tetragonum. Ocean Polar Res. 2011, 33, 185-191.

18. Park, J.H.; Li, C.; Hu, W.; Wang, M.H. Antioxidant and Free Radical Scavenging Activity of Different Fractions from Hawthorn Fruit. J. Food Sci. Nutr. 2010, 5, 44-50.

19. Hobbs, A.; Higgs, A.; Moncada, S. Inhibition of nitric oxide synthase as a potential therapeutic target. Ann. Rev. Pharm. Toxicol. 1999, 39, 191-220.

20. Alessandra, R.; Venera, C.; Laura, L.; Luca, V.; Angelo, V.; Juan, A.G. Antioxidant activity and antiproliferative action of methanolic extract of Geum quellyon Sweet roots in human tumor cell lines. J. Ethnopharmacol. 2005, 100, 323-332.

21. Hatano, T.; Edamatsu, R.; Hiramatsu, M.; Mori, A.; Fujita, Y.; Yasuhara, T. Effects of the interaction of tannins with co-exist substances. IV. Effects of tannins and ralated polyphenols on superoxide anion radical, and on 1,1-diphenyl-2-picrylhydrazyl radical. Chem. Pharm. Bull. 1989, 37, 2016-2021.

22. Christin, A.; Martine, T.; Jacques, H. Superoxide anion involvement in NBT reduction by NADPH-cytochrome P-450 reductase. FEBS Lett. 1978, 89, 26-28.

23. Toshiyuki, F.; Hideyuki, I.; Takashi, Y. Antioxidative polyphen-ols from walnuts (Juglans regia L.). Phytochemistry 2003, 63, 795-801.

24. Mosmann, T. Rapid colorimetric assay for the cellular growth and survival. J. Immunol. Methods 1983, 65, 55-63.

25. Yi, Z.C.; Liu, Y.Z.; Li, H.X.; Yin, Y.; Zhuang, F.Y.; Fan, Y.B.; Wang, Z. Tellimagrandin I enhances gap junctional communication and attenuates the tumor phenotype of human cervical carcinoma HeLa cells in vitro. Cancer Lett. 2006, 242, 77-87.

26. Miyamoto, K.; Nomura, M.; Murayama, T.; Furukawa, T.; Hatano, T.; Yoshida, T.; Koshiura, R.; Okuda, T. Antitumor activities of ellagitannins against sarcoma-180 in mice. Biol. Pharm. Bull. 1993, 16, 379-387.

27. Ken, S.F. Recent progress in ellagitannin chemistry. Phytochemistry 2005, 66, 1984-2000.

Sample Availability: Samples of the compounds 1-15 are available from the authors.

(C) 2013 by the authors; licensee MDPI, Basel, Switzerland. This article is an open access article distributed under the terms and conditions of the Creative Commons Attribution license (http://creativecommons.org/licenses/by/3.0/). 